\title{
Effect of Mandibular Advancement Splint on Obstructive Sleep Apnea with Insulin Resistant Diabetes
}

\author{
Ashutosh Gupta ${ }^{1}$, Arvind Tripathi ${ }^{1}$, Praveen Rai ${ }^{1}$, Piyush Sharma ${ }^{2}$, Vijay Yadav $^{1}$, Dewanshu Kumar ${ }^{3}$ \\ ${ }^{1}$ Department of Prosthodontics, Saraswati Dental College, Lucknow, \\ ${ }^{2}$ Department of Orthodontics, Dental College Azamgarh, Azamgarh, \\ ${ }^{3}$ Department of Prosthodontics, Maharana Pratap Dental College, Kanpur, India
}

Received February 28, 2020

Revised March 31, 2020

Accepted April 27, 2020

Address for correspondence Praveen Rai, MDS

Department of Prosthodontics, Saraswati Dental College,

233 Tiwari ganj,

Lucknow 227105, India

Tel: +91-9140523318

Fax: +91-9793325773

E-mail: drpraveenmdsprostho@ gmail.com
Objectives: Obstructive Sleep apnea (OSA) is characterized by complete or partial obstruction of upper airflow despite the effort to breathe, leading to hypoxemia and hypercapnia. The resultant apnea causes sleep fragmentation, which in turn increases sympathetic activity, decreases insulin sensitivity and glucose uptake, and stimulates hepatic gluconeogenesis that ultimately leads to type 2 diabetes. Most studies exploring the effect of continuous positive airway pressure on insulin sensitivity have showed a positive effect. However, there is no evidence on the effect of mandibular advancement device on insulin resistance (IR). This study was aimed to evaluate the effect of mandibular advancement splint (MAS) on IR in patients with OSA. Methods: The present study was conducted at Department of Prosthodontics, Dental College Azamgarh, from June 2015 to July 2017. Sixty eight dentulous patients with type 2 diabetes and mild to moderate OSA and with stable diabetic regimen were included in the study. A MAS was fabricated and fixed at $70 \%$ of the maximum mandibular protrusion recorded. Patients that were comfortable with MAS after one month were assessed for apnea-hypopnea index, mean oxygen saturation, and IR at baseline, 6 months, and 1 year after wearing MAS. Results: An improvement in insulin sensitivity was observed at 6 months for mild OSA patients $(p=0.001)$. For moderate OSA patients, no significant improvement was observed following MAS use $(p>0.05)$. Conclusions: The finding suggested that MAS is effective in improving IR in mild OSA patients.

J Sleep Med 2020;17(1):44-48

Key Words: Apnea-hypopnea index, Mandibular advancement splints, Obstructive sleep apnea, Insulin resistance.

\section{Introduction}

Obstructive sleep apnea (OSA) is a common affliction caused by intermittent obstruction of the upper respiratory tract. Despite the effort to breathe, there are repeated cessations of normal breathing. These cessations are termed as apneas. The resultant apnea causes sleep fragmentation, which in turn increases sympathetic activity, decreases insulin sensitivity and glucose uptake, and stimulates hepatic gluconeogenesis that ultimately leads to type 2 diabetes. ${ }^{1}$ Type 2 diabetes is a major chronic disease with high morbidity, mortality, and economic burden. ${ }^{2}$ Obesity, one of the prime reason for the alarming rise in the prevalence of type 2 diabetes. ${ }^{3,4}$ However, recent

This is an Open Access article distributed under the terms of the Creative Commons Attribution Non-Commercial License (https://creativecommons.org/licenses/by-nc/4.0) which permits unrestricted non-commercial use, distribution, and reproduction in any medium, provided the original work is properly cited. evidence based, clinical based, and laboratory based studies suggested that diabetes and OSA are independently associated with degree of adiposity. ${ }^{4}$ A few laboratory studies in healthy young subjects have found that, under well-controlled conditions, restricting sleep duration had an adverse impact on glucose tolerance. ${ }^{5,6}$ These studies suggest that treatment of OSA condition will ultimately improve glucose metabolism and eventually will lead to improved glucose control, however the effect of OSA treatment on glucose control has been variable.

Review of the literature found that most studies exploring the effect of continuous positive airway pressure (CPAP) on insulin sensitivity show a positive effect, ${ }^{3}$ however literature lack evidences on the effect of mandibular advancement device (MAD) on insulin resistance (IR). Hence the present study was planned to evaluate the effect of mandibular advancement splint (MAS) on IR in patients with OSA. 


\section{Methods}

The present study was conducted at Department of Prosthodontics, Dental College Azamgarh, from June 2015 to July 2017. Ethical approval was obtained from Committee for Ethics in Human Research Azamgarh. All the patients were informed about the nature of the study and written informed consent was taken from all the patients.

The inclusion criteria were as follows: 1) OSA patients with type 2 diabetes mellitus with stable diabetic control under oral hypoglycaemic medication (sulfonylurea or alpha-glucosidase inhibitor), 2) $\mathrm{HbAlc}$ value $>5.8 \%$ since in India the cutoff value of $\mathrm{HbAlc}$ for the patient to have diabetes is $>5.8 \%$, 3) age range 35 to 50 years, and 4) non obese (body mass index $\left.18-25 \mathrm{~kg} / \mathrm{m}^{2}\right)$. The exclusion criteria were as follows: 1) patients on insulin therapy, 2) apnea-hypopnea index (AHI) $>30$ since oral appliances are more effective in mild and moderate OSA, 3) craniofacial abnormalities (cleft lip and palate, retruded mandible) since these might act as confounding factors by further accentuating the condition of OSA, and 4) mobile tooth and temporomandibular joint disorders including pain, significant joint crepitation, restricted mouth opening, and masticatory muscle tenderness since they effect the normal functioning of MAS.

The primary screening yielded a cohort 92 patients who were assessed for excessive daytime sleepiness and sleep disordered breathing by the Epworth Sleepiness Scale and Berlin Questionnaire, respectively. 86 symptomatic patients were advised to undergo all night polysomnography. Based on AHI, patients were divided into mild ( $\mathrm{n}=28)$, moderate $(\mathrm{n}=44)$, and severe $(n=14)$ grade of OSA. Severe grade OSA patients were excluded from the study. For the remaining patients $(n=72)$, a MAS was fabricated and was fixed at $70 \%$ of the maximum mandibular protrusion recorded, to avoid any possible anteri-

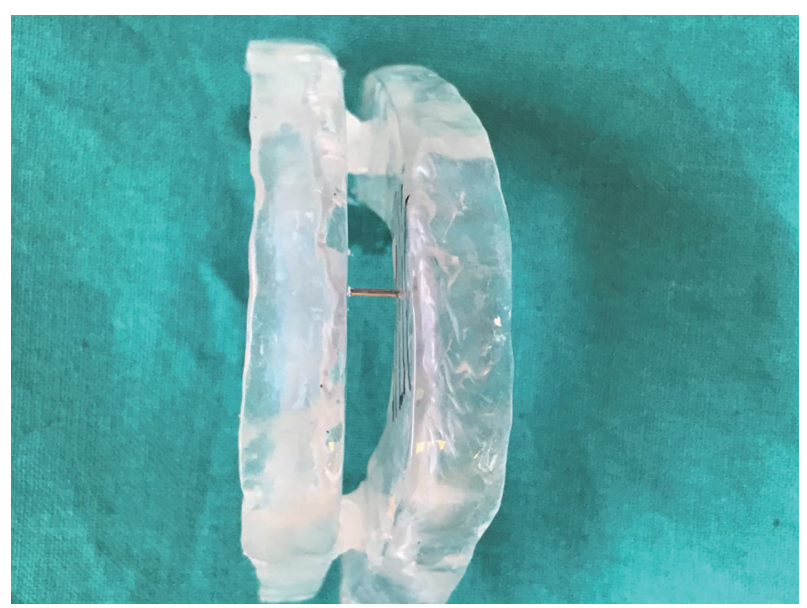

Figure 1. Mandibular advancement splint. or impingement of the glenoid fossae by the condyles (Fig. 1). All patients were recalled every week till one month. Four patients did not report. Patients that were comfortable with MAS after one month $(\mathrm{n}=68)$ were assessed for AHI, mean oxygen $\left(\mathrm{O}_{2}\right)$ saturation and IR at baseline, 6 months, and 1 year after wearing MAS. Thus the sample size constituted twenty eight mild OSA patients and forty moderate OSA patients. A self reported adherence to the oral appliance therapy was assessed every week via telephone. No patients were lost in follow-up. Demographic data is presented in Table 1.

\section{$\mathrm{AHI}$ and mean $\mathrm{O}_{2}$ saturation}

A full night polysomnography was performed in the sleep laboratory by sleep technician using S-7000 computerized polysomnography machine (Cogent Technologies, Woodbridge, U.K.) that included electroencephalograms, electrooculogram, chin and leg electromyogram, nasal airflow (nasal pressure cannula), $\mathrm{O}_{2}$ saturation (pulse oximetry), movements of thorax and abdomen, electrocardiogram, and body position. Somnologica Studio software (Embala corporation, Thornton, CO, USA) was used to calculate AHI in accordance with American Academy of Sleep Medicine manual for the scoring of sleep and associated events, version 2.0. ${ }^{9}$ AHI was determined by the frequency of these events per hour during sleep time based on the results of the overnight polysomnography. ${ }^{8}$

\section{IR}

Venous blood was sampled in the morning after an overnight fast for the measurement of the values of plasma glucose, serum insulin, and $\mathrm{HbAlc}$. Plasma glucose was measured by glucose oxidase method, serum insulin was measured with radioimmunoassay, and $\mathrm{HbAlc}$ values were measured by high performance liquid chromatography. Homeostasis model assessment (HOMA) was used to estimate IR. ${ }^{10}$ HOMA-IR was calculated with the formula: fasting plasma glucose $(\mathrm{mmol} / \mathrm{L})$ times fasting serum insulin (mU/L) divided by 22.5 . Low HOMA-IR values indicate high insulin sensitivity, whereas high HOMA-IR values indicate low insulin sensitivity. The normal range of HOMA-IR value is $0.5-1.4$ in healthy individual. HOMA-IR value above 2.9 indicates significant IR. ${ }^{11,12}$

Table 1. Demographic characteristics of the patients

\begin{tabular}{lcclcc}
\hline & \multicolumn{2}{c}{ Mild OSA } & & \multicolumn{2}{c}{ Moderate OSA } \\
\cline { 2 - 3 } \cline { 5 - 6 } & Baseline & 1 year & & Baseline & 1 year \\
\hline Age (years) & $42 \pm 6$ & $43 \pm 6$ & & $42 \pm 6$ & $43 \pm 6$ \\
Sex (male/female) & $20 / 8$ & $20 / 8$ & & $28 / 12$ & $28 / 12$ \\
BMI $\left(\mathrm{kg} / \mathrm{m}^{2}\right)$ & $22 \pm 5$ & $21.5 \pm 2.2$ & & $22 \pm 5$ & $21.5 \pm 2.2$ \\
HbA1c $(\%)$ & $6.2 \pm 0.02$ & & & $6.1 \pm 0.03$ & \\
\hline
\end{tabular}

OSA: obstructive sleep apnea, BMI: Body Mass Index 
Data was analyzed using Statistical Package for Social Sciences Version 24.0 (IBM Corp., Armonk, NY, USA). Analysis of variance statistical analysis was done with paired t-test to assess the significance of mean difference at different time points within each OSA severity sub-group for all three parameters (AHI, mean $\mathrm{O}_{2}$ saturation, and HOMA-IR value). Pearson correlation analysis was used to correlate between AHI and other parameters. A $p$ value less than 0.05 indicated statistically significant difference.

\section{Results}

The baseline, 6 months, and 1 year AHI scores, mean $\mathrm{O}_{2}$ saturation, and HOMA-IR values are given in Table 2. A statistically significant reduction in mean AHI was observed after 6 months and 1 year of wearing oral appliance $(p=0.051$ and $p<$ 0.01 , respectively) in both mild and moderate OSA patients.

No statistically significant improvement in mean $\mathrm{O}_{2}$ saturation was observed after 6 months of MAS use ( $p=0.061$ for mild OSA patients and $p=0.072$ for moderate OSA patients); however long term use (1 year) of MAS showed a statistically significant improvement in mean $\mathrm{O}_{2}$ saturation in both mild as well as moderate OSA patients $(p=0.05$ and $p=0.002$, respectively).

For mild OSA patients a statistically significant reduction in HOMA-IR value was observed at 6 months and 1 year $(p=$ 0.001 and $p=0.002$, respectively); however for moderate OSA patients no significant improvement was observed following MAS use ( $p=0.306$ at 6 months and $p=0.172$ at 1 year).

On seeking the correlation between $\mathrm{AHI}$, mean $\mathrm{O}_{2}$ satura- tion, and HOMA-IR value at 6 months, only HOMA-IR value showed a significant positive correlation with AHI ( $\mathrm{r}=0.547$; $p=0.03$ ) in mild OSA patients (Table 3). No significant correlation was observed for moderate OSA patients (Table 3). At 1 year, HOMA-IR value showed a moderate positive correlation with $\mathrm{AHI}$ in mild as well as moderate OSA patients ( $\mathrm{r}=$ $0.522, p=0.04 ; \mathrm{r}=0.445, p=0.03$, respectively) (Table 4 ).

\section{Discussion}

The present pilot study was aimed at evaluating the effect of oral appliance on IR on a cohort of OSA patients with type 2 diabetes and who were not on insulin therapy and had stable diabetic regime. We hypothesized that MAS will not have any effect on insulin sensitivity in OSA patients.

The results of the study indicated that 6 months of MAS use significantly decreases IR in mild OSA patients. No significant improvement in insulin sensitivity was observed in moderate OSA patients with 6 months use of oral appliance; however long term use showed significant improvement in IR. This might be due to the effectiveness of oral appliance in treating mild OSA patients as compared to moderate OSA patients. ${ }^{13-15}$ Although there was improvement in HOMA-IR value but there was no normalisation. ${ }^{11}$ The maximum improvement was observed in mild OSA group that used MAS for 1 year. Bleifeld et al. showed that the longer the duration of CPAP use, the better was the insulin sensitivity. ${ }^{16}$ Similar observation was reported by Harsch et al. that treatments that improve OSA will also improve insulin sensitivity in diabetic subjects with OSA, ${ }^{17}$ although this improvement seems to take

Table 2. Effect of mandibular advancement splint on $\mathrm{AHI}$, mean $\mathrm{O}_{2}$ saturation, and insulin resistance at 6 months and 1 year

\begin{tabular}{|c|c|c|c|c|c|c|c|c|c|c|}
\hline \multirow[b]{2}{*}{ Parameters } & \multicolumn{5}{|c|}{ Mild OSA } & \multicolumn{5}{|c|}{ Moderate OSA } \\
\hline & Baseline & $\begin{array}{l}6 \text { months } \\
\text { after } \\
\text { MAS use }\end{array}$ & $p$ & $\begin{array}{c}1 \text { year } \\
\text { after } \\
\text { MAS use }\end{array}$ & $p$ & Baseline & $\begin{array}{c}6 \text { months } \\
\text { after } \\
\text { MAS use }\end{array}$ & $p$ & $\begin{array}{c}1 \text { year } \\
\text { after } \\
\text { MAS use }\end{array}$ & $p$ \\
\hline AHI & $17.40 \pm 1.05$ & $5.53 \pm 2.64$ & 0.051 & $4.80 \pm 2.11$ & 0.05 & $24.15 \pm 3.04$ & $6.00 \pm 1.74$ & $<0.001$ & $6.00 \pm 1.74$ & 0.004 \\
\hline Mean $\mathrm{O}_{2}$ saturation & $93.12 \pm 0.70$ & $94.96 \pm 1.24$ & 0.061 & $95.36 \pm 0.88$ & 0.05 & $92.40 \pm 1.43$ & $93.68 \pm 1.48$ & 0.072 & $93.95 \pm 1.29$ & 0.002 \\
\hline HOMA & $4.85 \pm 0.39$ & $4.50 \pm 0.44$ & 0.001 & $4.37 \pm 0.45$ & 0.002 & $5.63 \pm 0.36$ & $4.13 \pm 0.59$ & 0.306 & $4.05 \pm 0.54$ & 0.172 \\
\hline
\end{tabular}

AHI: apnea-hypopnea index, $\mathrm{O}_{2}$ : oxygen, OSA: obstructive sleep apnea, MAS: mandibular advancement splint, HOMA: homeostasis model assessment

Table 3. Correlation (after 6 months) between $\mathrm{AHI}$, mean $\mathrm{O}_{2}$ saturation, and HOMA-IR values in patients with mild and moderate OSA

\begin{tabular}{lccccccc}
\hline & \multicolumn{3}{c}{ Mild OSA } & & \multicolumn{3}{c}{ Moderate OSA } \\
\cline { 2 - 3 } & AHI & Mean $\mathrm{O}_{2}$ saturation & HOMA-IR & & AHI & Mean $\mathrm{O}_{2}$ saturation & HOMA-IR \\
\hline AHI & - & & & - & - & \\
Mean $\mathrm{O}_{2}$ saturation & -0.222 & - & & -0.260 & - & - \\
HOMA-IR & $0.547^{*}$ & -0.119 & & & 0.271 & -0.024 & - \\
\hline
\end{tabular}

*correlation is significant at 0.05 level (two- tailed). AHI: apnea-hypopnea index, $\mathrm{O}_{2}$ : oxygen, HOMA: homeostasis model assessment, IR: insulin resistance, OSA: obstructive sleep apnea 
Table 4. Correlation (after 1year) between $\mathrm{AHI}$, mean $\mathrm{O}_{2}$ saturation, and HOMA-IR values in patients with mild and moderate OSA

\begin{tabular}{|c|c|c|c|c|c|c|}
\hline & \multicolumn{3}{|c|}{ Mild OSA } & \multicolumn{3}{|c|}{ Moderate OSA } \\
\hline & AHI & Mean $\mathrm{O}_{2}$ saturation & HOMA-IR & AHI & Mean $\mathrm{O}_{2}$ saturation & HOMA-IR \\
\hline AHI & - & & & - & & \\
\hline Mean $\mathrm{O}_{2}$ saturation & -0.241 & - & & -0.051 & - & \\
\hline HOMA-IR & $0.522^{*}$ & -0.196 & - & $0.445^{*}$ & -0.075 & - \\
\hline
\end{tabular}

*correlation is significant at 0.05 level (two- tailed). AHI: apnea-hypopnea index, $\mathrm{O}_{2}$ : oxygen, HOMA: homeostasis model assessment, IR: insulin resistance, OSA: obstructive sleep apnea

somewhat longer than in nondiabetic subjects. Wang and Wei et al. showed that short term use of CPAP could improve the hypoxia of the patients and it could enhance the insulin sensitivity. ${ }^{18,19}$ Although CPAP is the gold standard for treating OSA, effectiveness of oral appliance in mild OSA has also been established.

Another finding of the study was reduction in $\mathrm{AHI}$ and improvement in mean $\mathrm{O}_{2}$ saturation in mild and moderate OSA patients. The most commonly employed measure of OSA is AHI. Our findings are consistent with Gao et al. who found that after intervention by MAD, AHI decreased significantly. ${ }^{20}$ Our findings agree with those of Liu et al. and Ranieri et al. who found statistically significant reduction in AHI score after MAD intervention. ${ }^{21,22}$ Studies have observed that a reduction in AHI score was related to an increased velopharynx cross-sectional area after use of MAD.

Thus our hypothesis was rejected as there was the improvement in insulin sensitivity in OSA patients after intervention with MAS. This study provides evidence to inform health care worker about possible use of MAS in OSA with type 2 diabetes mellitus. To our knowledge, this study is the first to objectively evaluate the oral appliance effect on IR. Limitations of the present study include absence of any controls that would have correlated between normal OSA patients and patients with type 2 diabetes mellitus. Another limitation was Somnologica Studio software was used to calculate AHI without being reviewed by a physician. Although there are studies predicting the reliability of automated scoring of polysomnographic data over manual scoring, ${ }^{23-25}$ further studies are required before using this software alone. Another limitation was the effect of lifestyle factors and obesity, which were not part of the study.

In conclusion, the finding suggested that oral appliance is effective in improving IR in mild OSA patients. The severity of OSA has been associated with IR and subsequent impaired glucose metabolism, however further research in this field is warranted.

\section{Acknowledgments}

None.

\section{Conflicts of Interest}

The authors have no potential conflicts of interest to disclose.

\section{ORCID iDs}

$\begin{array}{ll}\text { Ashutosh Gupta } & \text { https://orcid.org/0000-0001-9064-5993 } \\ \text { Arvind Tripathi } & \text { https://orcid.org/0000-0003-0924-3407 } \\ \text { Praveen Rai } & \text { https://orcid.org/0000-0002-8814-1312 } \\ \text { Piyush Sharma } & \text { https://orcid.org/0000-0003-2038-6783 } \\ \text { Vijay Yadav } & \text { https://orcid.org/0000-0002-5100-0778 } \\ \text { Dewanshu Kumar } & \text { https://orcid.org/0000-0002-5438-0622 }\end{array}$

\section{Author Contributions}

Conceptualization: Ashutosh Gupta, Arvind Tripathi, Praveen Rai. Data curation: Ashutosh Gupta, Piyush Sharma, Vijay Yadav. Formal analysis: Ashutosh Gupta, Dewanshu Kumar. Investigations: Ashutosh Gupta. Methodology: Ashutosh Gupta, Arvind Tripathi, Praveen Rai. Supervision: Arvind Tripathi. Writing_original draft: Ashutosh Gupta, Arvind Tripathi. Writing_-review \& editing: Ashutosh Gupta, Praveen Rai, Piyush Sharma.

\section{REFERENCES}

1. Stamatakis KA, Punjabi NM. Effects of sleep fragmentation on glucose metabolism in normal subjects. Chest 2010;137:95-101.

2. Tasali E, Mokhlesi B, Van Cauter E. Obstructive sleep apnea and Type 2 diabetes: interacting epidemics. Chest 2008;133:496-506.

3. Nannapaneni S, Ramar K, Surani S. Effect of obstructive sleep apnea on Type 2 diabetes mellitus: a comprehensive literature review. World J Diabetes 2013;4:238-244.

4. Botros N, Concato J, Mohsenin V, Selim B, Doctor K, Yaggi HK. Obstructive sleep apnea as a risk factor for Type 2 diabetes. Am J Med 2009; 122:1122-1127.

5. Mallon L, Broman JE, Hetta J. High incidence of diabetes in men with sleep complaints or short sleep duration: a 12-year follow-up study of a middle-aged population. Diabetes Care 2005;28:2762-2767.

6. Spiegel K, Leproult R, Van Cauter E. Impact of sleep debt on metabolic and endocrine function. Lancet 1999;354:1435-1439.

7. Nair M, Prabhakaran D, Narayan KM, et al. $\mathrm{HbA}(1 \mathrm{c})$ values for defining diabetes and impaired fasting glucose in Asian Indians. Prim Care Diabetes 2011;5:95-102.

8. Gupta A, Tripathi A, Sharma P. The long-term effects of mandibular advancement splint on cardiovascular fitness and psychomotor performance in patients with mild to moderate obstructive sleep apnea: a prospective study. Sleep Breath 2017;21:781-789.

9. Berry RB, Budhiraja R, Gottlieb DJ, et al. Rules for scoring respiratory events in sleep: update of the 2007 AASM manual for the scoring of sleep and associated events. Deliberations of the sleep apnea definitions task force of the American Academy of Sleep Medicine. J Clin Sleep Med 2012;8:597-619.

10. Matthews DR, Hosker JP, Rudenski AS, Naylor BA, Treacher DF, Turner RC. Homeostasis model assessment: insulin resistance and betacell function from fasting plasma glucose and insulin concentrations in man. Diabetologia 1985;28:412-419. 
11. Tang Q, Li X, Song P, Xu L. Optimal cut-off values for the homeostasis model assessment of insulin resistance (HOMA-IR) and pre-diabetes screening: developments in research and prospects for the future. Drug Discov Ther 2015;9:380-385.

12. Gayoso-Diz P, Otero-Gonzalez A, Rodriguez-Alvarez MX, et al. Insulin resistance index (HOMA-IR) levels in a general adult population: curves percentile by gender and age. The EPIRCE study. Diabetes Res Clin Pract 2011;94:146-155.

13. Aarab G, Lobbezoo F, Hamburger HL, Naeije M. Effects of an oral appliance with different mandibular protrusion positions at a constant vertical dimension on obstructive sleep apnea. Clin Oral Investig 2010; 14:339-345.

14. Bloch KE, Iseli A, Zhang JN, et al. A randomized, controlled crossover trial of two oral appliances for sleep apnea treatment. Am J Respir Crit Care Med 2000;162:246-251.

15. Sutherland K, Vanderveken OM, Tsuda H, et al. Oral appliance treatment for obstructive sleep apnea: an update. J Clin Sleep Med 2014;10: 215-227.

16. Bleifeld W. Fibrinolysis in acute myocardial infarct. Schweiz Med Wochenschr 1987;117:1641-1647.

17. Harsch IA, Konturek PC, Koebnick C, et al. Leptin and ghrelin levels in patients with obstructive sleep apnoea: effect of CPAP treatment. Eur Respir J 2003;22:251-257.

18. Wang H, Wang L, Liu J. The effect of short-time continuous positive airway pressure treatment on insulin sensitivity in patients with obstructive sleep apnea-hypopnea syndrome and Type 2 diabetes. Lin Chung Er
Bi Yan Hou Tou Jing Wai Ke Za Zhi 2008;22:597-599.

19. Wei CY, Wang HL, Li J, et al. Effect of continuous positive airway pressure upon $24 \mathrm{~h}$ changes of blood glucose level in patients with obstructive sleep apnea hypopnea syndrome and Type 2 diabetes. Zhonghua Yi Xue Za Zhi 2009;89:2686-2689.

20. Gao XM, Zeng XL, Fu MK, Huang XZ. Magnetic resonance imaging of the upper airway in obstructive sleep apnea before and after oral appliance therapy. Chin J Dent Res 1999;2:27-35.

21. Liu Y, Lowe AA, Fleetham JA, Park YC. Cephalometric and physiologic predictors of the efficacy of an adjustable oral appliance for treating obstructive sleep apnea. Am J Orthod Dentofacial Orthop 2001;120: 639-647.

22. Ranieri AL, Jales SM, Formigoni GG, de Alóe FS, Tavares SM, Siqueira JT. Treatment of obstructive sleep apnea syndrome in patients from a teaching hospital in Brazil: is it possible? Sleep Breath 2009;13:121-125.

23. Malhotra A, Younes M, Kuna ST, et al. Performance of an automated polysomnography scoring system versus computer-assisted manual scoring. Sleep 2013;36:573-582.

24. Ferri R, Ferri P, Colognola RM, Petrella MA, Musumeci SA, Bergonzi P. Comparison between the results of an automatic and a visual scoring of sleep EEG recordings. Sleep 1989;12:354-362.

25. Byun JI, Song SJ, Cha HK, Shin WC. Reliability of manual and automatic scoring of single channel Nasal Airflow Device (ApneaLink) in determining moderate or severe obstructive sleep apnea syndrome. Sleep Med Res 2016;7:68-73. 